Int. J. Odontostomat., 8(3):337-343, 2014.

\title{
Salud Oral en Adultos Mayores Postrados
}

\author{
Oral Health in Homebound Elderly Adults
}

María E. Quinteros* \& Loreto Nuñez Franz*

QUINTEROS M. E. \& NUÑEZ, F. L. Salud oral en adultos mayores postrados. Int. J. Odontostomat., 8(3):337-343, 2014.

RESUMEN: La población de adultos mayores ha aumentado en los últimos años y se hace imprescindible determinar su estado de salud oral con el objetivo de orientar la atención de salud con base científica epidemiológica para brindarles una mejor calidad de vida. El objetivo de este trabajo fue evaluar el estado de la salud oral de los pacientes adultos mayores postrados. Se realizó un estudio descriptivo de corte transversal no probabilístico. Los datos fueron obtenidos del universo de pacientes inscritos en el programa de Atención Domiciliaria del Paciente Postrado de un centro de atención primaria. La tasa de respuesta obtenida fue $90 \%$. El sexo predominante fue femenino; la media de edad fue 80 años; $60,9 \%$ sólo tuvo acceso a la educación primaria. La prevalencia de caries fue 100\% y COPD de 25,6. El 40,6\% se encontraba completamente edentulo y el $54,7 \%$ era portador de prótesis dental. El 32,8\% presentaba restos radiculares. El 64,7\% era capaz de cepillar sus dientes por sí mismo. El 79,4\% consideraba tener una pobre salud oral. El cuidador resultó ser la hija, de aproximadamente 53 años, con nivel educacional bajo. Los adultos mayores son un grupo con gran daño odontológico, con escasos dientes remanentes y deficientes prácticas de higiene oral. Son cuidados por familiares con bajo nivel educacional.

PALABRAS CLAVE: adulto mayor postrado, prevalencia, higiene oral, COPD, GOHAI.

\section{INTRODUCCIÓN}

Actualmente la población mundial crece con una tasa de $1,7 \%$ anual, mientras que el grupo mayor de 60 años lo hace con una tasa de $2,5 \%$ y sobre los 80 años con una tasa de $4 \%$. Aproximadamente $600 \mathrm{mi}-$ llones de personas están sobre los 60 años y más, y este número llegará al doble en 2025. Para el 2050, sería de 2 billones, de los cuales $80 \%$ viviría en países desarrollados (United Nations, 2009).

Producto principalmente de patologías crónicas, alrededor del $7 \%$ de los adultos mayores (AM) se encuentran con dependencia severa o postrados, es decir, no están capacitados para realizar alguna actividad básica debido a una patología base que los aqueja. Un paciente postrado o encamado es una persona dependiente para cualquier actividad básica, sin posibilidades de recuperación dada su enfermedad de base (García et al., 2002).

Las estadísticas muestran que hasta el $20 \%$ de los AM de países en vías de desarrollo tienen importantes dificultades en su desplazamiento y la mitad de ellos se encuentran en estado de postración. En Chile no existe aún consenso en cuanto a las cifras de pacientes postrados, se estima que $7 \%$ de los AM viven en dicha condición (Albala et al., 2005).

Los problemas de la boca son frecuentes en los pacientes postrados y sin duda contribuyen de forma importante a aumentar su malestar, aislamiento $y$ angustia. El aumento de la esperanza de vida ha creado cambios para la sociedad y la profesión dental. Junto con el aumento de la población envejecida también aumentan quienes son considerados frágiles y funcionalmente afectados, los que no pueden vivir en forma independiente o participar en la comunidad, es por esto que los cuidados de salud oral de las personas postradas pueden contribuir a mejorar su calidad de vida y la del cuidador otorgando cuidados paliativos (Henry \& Ceridan, 1994). Hasta la fecha se desconoce el estado de salud bucal de los AM postrados en Chile, es por esto que el objetivo de esta investigación fue evaluar su estado de salud oral y caracterizar socio demográficamente a su cuidador(a) de un Centro de Atención Primaria. 


\section{MATERIAL Y MÉTODO}

Se realizó un estudio transversal que incluyó el universo de pacientes AM inscritos en el Programa de Atención Domiciliaria del Paciente Postrado del Centro de Salud Dr. José Dionisio Astaburuaga de la ciudad de Talca, Chile entre los meses de junio y agosto de 2006. Se invitó a participar del estudio a los AM postrados, dependientes para cualquier actividad básica y a sus cuidadores. Quedaron excluidos aquellos AM postrados que presentaron discapacidad cognitiva o verbal, o se encontraron en estado pre-agónico, agónico o terminal.

El examen de la cavidad oral se realizó mediante inspección visual con instrumental de examen siguiendo las recomendaciones de la Organización Mundial de la Salud (WHO, 1997). Todos los participantes fueron examinados en sus domicilios con iluminación natural más el apoyo de una lámpara ligera portátil emisora de luz artificial corregida.

Durante la primera etapa del estudio, se realizó una revisión de las fichas clínicas del Programa de Atención Domiciliaria del Paciente Postrado recogiéndose datos de identificación. Luego, se realizaron visitas domiciliarias invitando a participar en la investigación mediante un consentimiento informado, siguiendo los preceptos de la Declaración de Helsinki. Previo al trabajo de campo, se realizó la calibración del observador obteniéndose un coeficiente Kappa de 0,97 para caries con un intervalo de confianza del 95\% (IC $95 \%$ ) de 0,86-0,99 y para el índice de higiene oral de 0,85 (IC 95\%, 0,7-0,99), que indica un alto grado de concordancia para ambos casos.

La variables estudiadas fueron sexo, edad, seguro de salud, nivel socioeconómico (Adimark, 2003), escolaridad, Índice de dientes cariados, obturados y perdidos (COPD) (WHO), presencia de restos radiculares, uso de prótesis dental, Índice de Higiene Oral Simplificado (IHOs), prácticas de higiene oral (participación activa o pasiva del paciente en el cepillado dental, frecuencia del cepillado, duración del cepillado y empleo dentífrico), Índice de Valoración de la Salud Oral Geriátrica (GOHAl) (Misrachi \& Espinoza, 2005). Las variables estudiadas en el cuidador fueron edad, sexo, parentesco con el AM, nivel socioeconómico y nivel educacional.

Este estudio fue aprobado por el Comité de Ética de la Universidad de Talca. Los participantes del estudio y sus cuidadores recibieron educación para la salud una vez terminada la evaluación, se entregó a cada participante implementos de aseo bucodental (cepillo de dientes, cepillo de prótesis, colutorios de clorhexidina $0,12 \%$, según situación oral), un folleto educativo con medidas recomendadas para la higiene oral en AM postrados. Los participantes que necesitaban atención de urgencia odontológica, de enfermería o médica, fueron reportados al responsable del programa del centro de salud.

Los datos fueron codificados e ingresados por un solo operador. Previo al análisis se realizó la validación de éstos. El análisis estadístico proporcionó información descriptiva acerca de la distribución de prevalencia de las caries dentales con relación a variables independientes seleccionadas (edad, sexo, seguro de salud, nivel educacional, nivel socioeconómico, prácticas de higiene, GOHAl, IHOs), su promedio y medidas de dispersión como la desviación estándar en variables cuantitativas continuas. El procesamiento y administración de datos se efectuó utilizando el programa estadístico SPSS (version 15).

\section{RESULTADOS}

La población inscrita en el programa de Atención Domiciliaria del Paciente Postrado del centro de atención primaria de salud en 2006 correspondió a 168 pacientes, de los cuales se excluyeron aquellos fallecidos, los que no cumplieron con los criterios de inclusión y aquellos imposibles de contactar (Fig. 1), quedando 64 AM participantes, obteniéndose una tasa de respuesta de $90 \%$.

Datos sociodemográficos. La distribución de la población por sexo fue de $59,4 \%$ mujeres y $40,6 \%$ hombres. La media de edad fue de 80 años $(D E=9,9)$. El $76,6 \%$ de los participantes era mayor de 75 años.

El $60,9 \%$ tuvo acceso a la educación primaria, $15,6 \%$ a la secundaria y $14,1 \%$ no recibió educación formal. Sólo 9,4\% cursó estudios superiores. La mayoría de las personas postradas pertenecían al seguro público de salud $(98,4 \%)$. Los datos reportan que el nivel socio - económico predominante fue el medio bajo (46,9\%), seguido por el nivel medio $(28,1 \%)$ (Tabla I). 


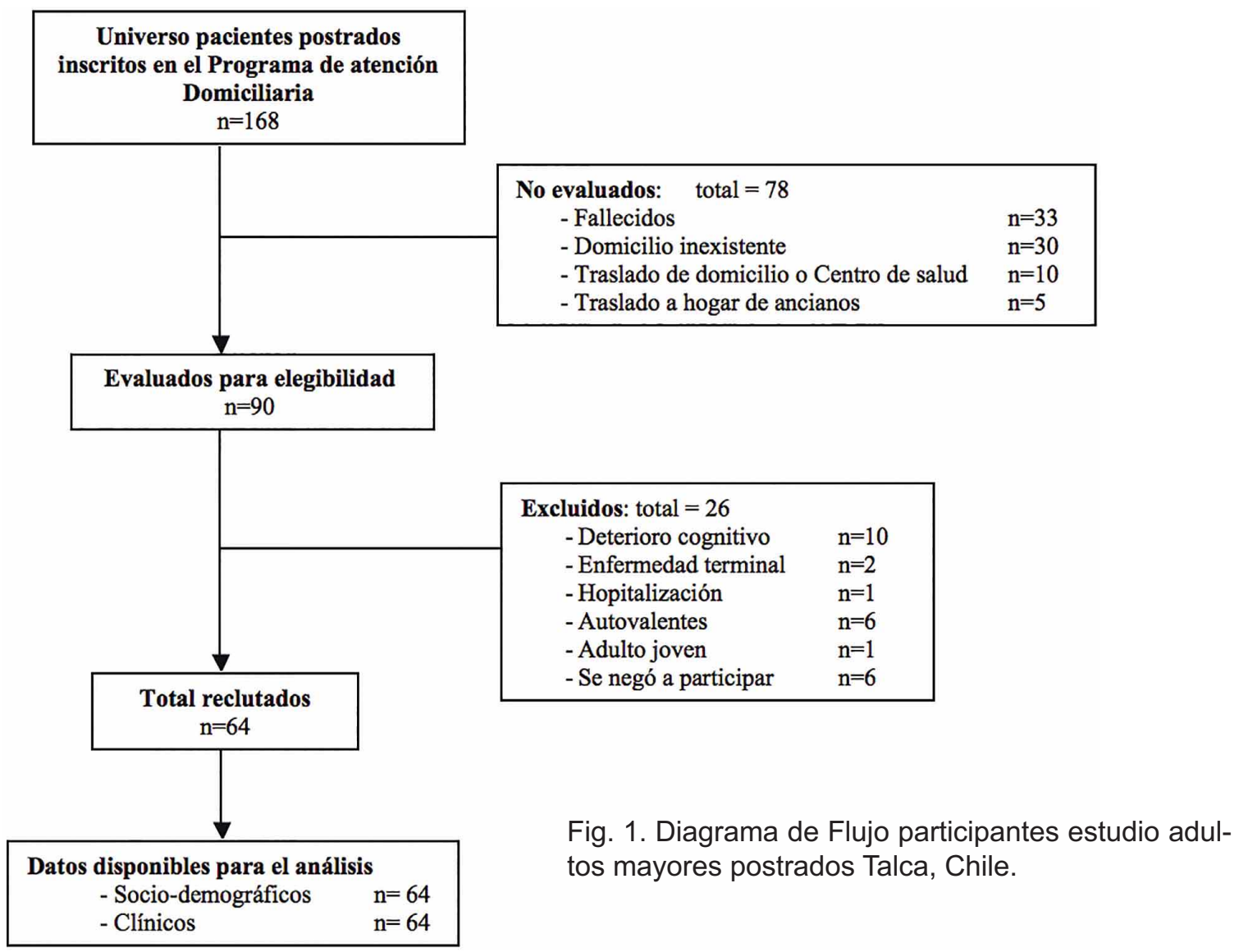

Tabla I. Distribución de la población de adultos mayores postrados según características socio-demográficas e historia de caries, Talca, Chile.

\begin{tabular}{|c|c|c|c|}
\hline \multirow{2}{*}{\multicolumn{2}{|c|}{ Variables }} & Adultos mayores & COPD \\
\hline & & $\mathrm{n}(\%)$ & Media (DE) \\
\hline \multicolumn{2}{|l|}{ Total participantes } & $64(100)$ & $25,6(3,3)$ \\
\hline \multicolumn{2}{|c|}{ Media de edad, años (DE) } & $80,02(9,9)$ & \\
\hline \multirow[t]{2}{*}{ Sexo } & Femenino & $38(59,4)$ & $24,8(3,8)$ \\
\hline & Masculino & $26(40,6)$ & $26,3(2,9)$ \\
\hline \multirow[t]{4}{*}{ Grupos de edad } & 55 a 64 años & $4(6,3)$ & $20,8(3,8)$ \\
\hline & 65 a 74 años & $11(17,2)$ & $24,9(4,2)$ \\
\hline & 75 a 84 años & $25(39,1)$ & $26,4(2,7)$ \\
\hline & 85 y más años & $24(37,5)$ & $26(2,9)$ \\
\hline \multirow[t]{4}{*}{ Escolaridad } & Sin educación & $9(14,1)$ & $27,7(1,0)$ \\
\hline & Primaria & $39(60,9)$ & $25,6(3,4)$ \\
\hline & Secundaria & $10(15,6)$ & $23,8(4,4)$ \\
\hline & Superior & $6(9,4)$ & $26,5(2,3)$ \\
\hline Nivel & Alto & - & or \\
\hline \multirow{4}{*}{ Socioeconómico } & Medio alto & $7(10,9)$ & $25,6(2,6)$ \\
\hline & Medio & $18(28,1)$ & $26,8(2,1)$ \\
\hline & Medio bajo & $30(46,9)$ & $24,9(4,0)$ \\
\hline & Bajo & $9(14,1)$ & $26,2(3,3)$ \\
\hline \multirow[t]{2}{*}{ Seguro de Salud } & Público & $61(98,4)$ & $25,7(3,4)$ \\
\hline & Privado & $1(1,6)$ & $28(0,0)$ \\
\hline
\end{tabular}


El 93,5\% de los cuidadores tenía una relación de parentesco con el AM postrado; $48,4 \%$ tenía como cuidador a su hija, $14,5 \%$ al cónyuge y $6,5 \%$ a la hermana. La media de edad del cuidador fue de 53,5 años $(D E=15,4)$. El nivel educacional predominante fue el secundario $(52,4 \%)$. El nivel socioeconómico dominante fue el medio bajo $(45,2 \%)$ (Tabla II).

Tabla II. Características socio-demográficas del cuidador de los adultos mayores postrados, Talca, Chile.

\begin{tabular}{llr}
\hline Variables & & \multicolumn{1}{c}{ Cuidador } \\
\cline { 3 - 3 } & & \multicolumn{1}{c}{$(\mathrm{n}=62)$} \\
\hline Sexo & Femenino, $n(\%)$ & $38(61,3)$ \\
Media de edad, años (DE) & Masculino, $n(\%)$ & $24(38,7)$ \\
Escolaridad & & $53,5(15,4)$ \\
& Sin educación formal, $n(\%)$ & $3(4,8)$ \\
& Primaria, $n(\%)$ & $19(30,2)$ \\
& Secundaria, $n(\%)$ & $33(52,4)$ \\
Nivel & Superior, $n(\%)$ & $8(12,6)$ \\
Socioeconómico & Alto, $n(\%)$ & $0(0,0)$ \\
& Medio alto, $n(\%)$ & $7(11,3)$ \\
& Medio, $n(\%)$ & $18(29,0)$ \\
& Medio bajo, $n(\%)$ & $28(45,2)$ \\
& Bajo, $n(\%)$ & $9(14,5)$ \\
\hline
\end{tabular}

Estado oral. La prevalencia de caries correspondió al 100\%, con un COPD de 25,6 (DE=3,3). Se observa una media de COPD más alto en los hombres $(24,8 ; \mathrm{DE}=2,9)$ que en las mujeres $(24,8 ; \mathrm{DE}=3,8)$. En los participantes mayores de 75 años se observan valores de COPD mayores en comparación con los otros grupos. El mayor daño oral se constata en las personas que no recibieron educación formal $(27,1 ; \mathrm{DE}=1,0)$; y que pertenecían al nivel socioeconómico medio $(26,8 ; \mathrm{DE}=2,1)$ (Tabla I). El $32,8 \%$ de los examinados presentaba restos radiculares en boca.

El $40,6 \%$ de la población se encontraba completamente edéntula, mientras que el $59,4 \%$ parcialmente. Un $54,7 \%$ era portador de prótesis (Tabla III).

Tabla III. Edentulismo y empleo de prótesis dentales en los adultos mayores postrados, Talca, Chile.

\begin{tabular}{llc}
\hline & & Adultos mayores \\
\hline Edentulismo & Completamente edentulos, $n$ & $26(40,6)$ \\
& Pacialmente edentulos, $\mathrm{n}(\%)$ & $38(59,4)$ \\
Usuario & Si, $n(\%)$ & $35(54,7)$ \\
Prótesis & No, $n(\%)$ & $28(43,8)$ \\
\hline
\end{tabular}

Prácticas de higiene oral. El $82,8 \%$ de los participantes cepillaba sus dientes. El 35,9\% de los AM se cepillaban una vez al día, $25,0 \%$ dos veces, $14,1 \%$ más de 2 veces y un $7,8 \%$ menos de una vez. El $67,9 \%$ era capaz de cepillar sus dientes por sí mismo, mientras que $32,1 \%$ requería de asistencia por parte del cuidador. El $71,7 \%$ cepi- llaba sus dientes menos de 3 minutos y $28,3 \%$ por más de 3 minutos. El $84,9 \%$ de los AM empleaba dentífrico (Tabla IV).

El índice de higiene oral de los participantes reveló que $88,9 \%$ se encontraba en riesgo de desarrollar caries y $11,1 \%$ sin riesgo. Este indicador reportó una media de 2,23 ( $D E=0,62)$, lo que se clasifica como una limpieza oral deficiente (Tabla IV).

Autopercepción de salud oral. Respecto a la autopercepción de salud oral, el $79,4 \%$ de los participantes consideraba tener una pobre salud oral, $11,8 \%$ moderada y $9,4 \%$ una buena salud oral. EI GOHAl obtuvo una media de 42,12 $(D E=10,72)$ lo que clasifica como baja, lo que indica una pobre autopercepción de la salud oral (Tabla IV).

\section{DISCUSIÓN}

Los datos de la investigación señalan que un mayor porcentaje de los adultos mayores postrados pertenecieron al sexo femenino $(61,3 \%)$ lo que concuerda con la realidad nacional, en la cual un $56,7 \%$ de los adultos mayores en el país son mujeres (MIDEPLAN, 2009) y con un estudio realizado en adultos mayores dependientes con pensión asistencial donde el $81 \%$ de los participantes fueron mujeres (SENAMA, 2007). El predominio del sexo femenino podría atribuirse a la mayor sobrevivencia de las mujeres ya que consultan en forma precoz y en mayor frecuencia al médico (Nathanson, 1975), además de la mayor esperanza de vida que presentan en Chile (82 años) en comparación con los hombres (76 años) (INE, 2008).

Los participantes pertenecían mayoritariamente al seguro público de salud $(98,4 \%)$, patrón de aseguramiento común en Chile $(88,12 \%)$ (MIDEPLAN). En el país con el paso de 
Tabla IV. Prácticas de higiene oral y autopercepción de la salud oral de los adultos mayores postrados, Talca, Chile.

\begin{tabular}{llr}
\hline & & Adulto mayor \\
\hline Frecuencia cepillado & No se cepilla, $n(\%)$ & $11(17,2)$ \\
& Menos de una vez al día, $n$ (\%) & $5(7,8)$ \\
& 1 vez al día, $n(\%)$ & $23(35,9)$ \\
& 2 veces al día, $n(\%)$ & $16(25,0)$ \\
Participación & Más de 2 veces al día, $n(\%)$ & $9(14,1)$ \\
cepillado & Activa, $n(\%)$ & $36(67,9)$ \\
Duración cepillado & Pasiva, $n(\%)$ & $17(32,1)$ \\
& Menos de 3 minutos, $n(\%)$ & $38(71,7)$ \\
Empleo dentífrico & Más de 3 minutos, $n(\%)$ & $15(28,3)$ \\
& Si, $n(\%)$ & $45(84,9)$ \\
Índice de Higiene Oral $(\%, n(\%)$ & $8(15,1)$ \\
& Non riesgo, $n(\%)$ & $24(88,9)$ \\
GOHAl & Sin riesgo, $n(\%)$ & $3(11,1)$ \\
& Pobre, $n(\%)$ & $27(79,4)$ \\
& Moderada, $n(\%)$ & $4(11,8)$ \\
& Buena, $n(\%)$ & $3(8,8)$ \\
\hline
\end{tabular}

los años se han instaurado políticas gubernamentales que brindan seguridad social a los adultos mayores, y que por supuesto ha beneficiado a este grupo de personas postradas quienes perciben jubilación, pensión o subsidios monetarios estatales (SENAMA, 2009), lo que les permite acceder en forma automática al seguro público de salud.

El nivel socioeconómico predominante fue el medio bajo; un porcentaje importante $(14,1 \%)$ pertenece al nivel bajo o de extrema pobreza lo que sumado al alto porcentaje que sólo accedió a educación primaria $(60,9 \%)$, son factores determinantes en el ingreso monetario de este grupo etario. Probablemente estos AM durante la etapa de la vida en que se encontraban económicamente activos, producto del bajo nivel educacional, su ingreso percibido también era bajo, imposibilitando generar ahorro y con ello niveles aceptables de seguridad económica (SENAMA, 2007). Esta situación dificultaría la compra de insumos médicos o de enfermería que son ampliamente empleados por este grupo.

El cuidador fue, en casi la mitad de la población, la hija del AM, similar a lo reportado en otro estudio donde en su mayoría el cuidador fue de sexo femenino (Morishita et al., 2001). El nivel educacional del cuidador fue secundario, implicando un cuidador con poca educación que podría dificultar la capacidad de mantener en las mejores condiciones posibles la salud general y oral de su familiar postrado.

En términos de la historia de caries, la pobla- ción estudiada había perdido dientes en el transcurso de la vida, en mayor o menor medida (COPD $=25,6$; $\mathrm{DE}=3,3$ ) lo que concuerda con estudios nacionales donde en el grupo de 65 a 74 años muestra valores COPD cercanos a 20 (Badenier, 2006). La prevalencia de caries fue de $100 \%$ que se explicaría por su condición de salud que les imposibilita el acceso a la atención odontológica y la entrega de medidas preventivas en sus hogares. Durante su vida este grupo etario tuvo poco acceso a las medidas promocionales y preventivas en salud bucodental, lo que conjugado con el bajo nivel socioeconómico, juegan roles relevantes en la etiopatogenia de la caries (Petersen et al., 2005). El 32\% de los participantes presentaba restos radiculares en boca, lo que se exacerbaría ante la nula posibilidad de recibir atención dental.

Los datos muestran que el 40,6\% era desdentado total y el $59,4 \%$ parcial, similar a los datos de la Encuesta Nacional de Salud en donde el $99 \%$ de los adultos mayores eran desdentados totales. Por otra parte un estudio realizado en Chile con adultos mayores autovalentes de 60 años de nivel socio-económico bajo del área norte de Santiago encontró que el $25,9 \%$ era desdentado total (Misrachi et al., 2002), dicha diferencia podría explicarse ya que en esa investigación los adultos mayores tenían menor edad que los pacientes postrados participantes de este estudio, lo que se asociaría al mayor número de años que tienen en promedio éstos últimos (80 años) y se evidenciaría un mayor daño a nivel del sistema estomatognático, traduciéndose en una mayor pérdida de dientes. 
Un $43,8 \%$ de los participantes no usaba prótesis dental lo que les produce dificultad para comer y procesar los alimentos, con las ya sabidas consecuencias negativas sobre su estado nutricional (Tada et al., 2002). Un estudio realizado en Escocia reporta que el $27 \%$ de la población no usaba prótesis (Manderson \& Ettinger, 1975), dicha diferencia se explicaría por el mayor acceso a atención odontológica que esa población tiene y por lo tanto una menor pérdida de dientes.

Las prácticas de higiene oral son deficientes, el $17,2 \%$ de los participantes no cepillaba sus dientes, cifra inferior a lo reportado en un estudio realizado en Japón donde el $46 \%$ de los individuos no cepillaba sus dientes (Morishita et al.). Esto podría atribuirse al bajo nivel educacional y a la alfabetización en salud buco oral. Se abre un espacio importante para la capacitación al paciente, su núcleo familiar y cuidador acerca de la importancia del cuidado oral.

La mayor parte de los adultos mayores $(79,4 \%)$ consideraba tener una pobre salud oral lo que se podría deber a que todos los pacientes en menor o mayor grado son desdentados lo que afecta la estética, función masticatoria o fonación; estas cifras han sido coincidentes con estudios chilenos (Albala et al.) y americanos (Kandelman et al., 1990; Paunovich, 1994) en donde el $73,5 \%$ y $84 \%$, respectivamente, de los adultos mayores consideró su salud oral como pobre. Subyacente a la problemática sobre la autoestima y la pobre autopercepción de salud oral, se suma además los problemas de malnutrición por déficit (Ritchie et al., 1997) que estos adultos mayores puedan estar sufriendo como consecuencia del desdentamiento.

Una fortaleza de esta investigación es el hecho de que sus resultados pueden generar otras investigaciones en este grupo, donde se aborde el rol del cuidador y el equipo de salud en el cuidado oral del adulto mayor postrado; realizar un estudio que compare la con- dición oral de los pacientes postrados institucionalizados, con la finalidad de gestar reformas de atención en salud para este grupo que incluya la atención odontológica móvil, terapias de restauración atraumáticas, resolución de urgencias y eliminación de sitios de infección o programa de rehabilitación con prótesis dentales. Hasta la fecha, los pacientes postrados son un grupo poblacional descuidado por la odontología, por lo que se es menester educar a esta población y a su familia, brindando apoyo adecuado al cuidador.

Una limitación del estudio es que no representa a los pacientes postrados que reciben atención domiciliaria en forma particular o institucionalizados, excluye a los pacientes con deterioro cognitivo que en este estudio resultó ser el $7 \%$ de la población estudiada. Por otra parte, ocurre en esta investigación un sesgo de recuerdo, ya que es sabido que en la edad adulta los pacientes pierden dientes también debido a enfermedad periodontal y es imposible determinar el real motivo de la indicación de una extracción.

En resumen, el AM postrado resultó ser mayoritariamente de sexo femenino, de edad avanzada, con un bajo nivel socioeconómico y educacional, perteneciente al seguro público de salud, cuyo cuidador fue principalmente la hija de nivel educacional bajo. La prevalencia de caries fue de 100\%, COPD de 25,6. Las prácticas de higiene fueron deficientes, con un alto riesgo cariogénico y una pobre autopercepción de su salud oral. Tras esta realidad descrita se torna imprescindible implementar de forma definitiva políticas de atención de salud para este grupo de personas, que hasta la fecha sólo dependen de la buena voluntad del equipo de salud, el cual debido a las exigencias de cumplimiento de metas les resulta cada vez más difícil llevar a cabo esta tarea.

AGRADECIMIENTOS. A los Dres. Rodrigo Giacaman Sarah y al Profesor Anish Koshi.

QUINTEROS, M. E. \& NUÑEZ, F. L. Oral health in homebound elderly adults. Int. J. Odontostomat., 8(3):337-343, 2014.

ABSTRACT: The elderly population is on an increase and it is important for us to know the status of their oral health in order to direct our attention to epidemiological aspects on a scientific basis to provide a better quality of life. The purpose of this study was to evaluate the status of oral health of the homebound elderly. A Cross sectional study was conducted using a non-probability sampling method. The data was collected from bedridden patients enrolled in a Patient Home Care in a Primary Care Center. The response rate obtained was $90 \%$. The majority of patients were female; their average age was 80 . Only $60.9 \%$ of those surveyed had access to primary education. The prevalence of caries was $100 \%$ and the DMFT index was $25.6 ; 40.6 \%$ were edentulous and $54.7 \%$ had dentures. The $32.8 \%$ had root fragments; $82.8 \%$ were able to brush their teeth by themselves. $79.4 \%$ were observed to have poor oral health. In almost all cases, the caretaker was a daughter around 53 years of age, with low education level. The group surveyed had significant dental damage, had only a few remaining teeth and observed poor oral hygiene practices. Their care was also almost exclusively in the hands of family members who had a low education level.

KEY WORDS: homebound elderly, prevalence, oral hygiene, DMFT, GOHAI. 


\section{REFERENCIAS BIBLIOGRÁFICAS}

Adimark, Mapa Socio-económico de Chile. Nivel socioeconómico de los hogares del país basado en datos del Censo. Santiago, Adimark, 2003. Disponible en: www.adimark.cl

Albala, C.; Lebrão, M. L.; León Díaz, E. M.; Ham-Chande, R.; Hennis, A. J.; Palloni, A.; Peláez, M. \& Pratts, O. Encuesta de Salud, Bienestar y Envejecimiento (SABE): metodología de la encuesta y perfil de la población estudiada. Rev. Panam. Salud Publica, 17(5-6):307-22, 2005.

Badenier, O.; Moya, R. \& Cueto, A. Estudio de prevalencia de Enfermedades Bucodentales y necesidades de tratamiento en la $V$ región. Universidad de Valparaíso, Chile. Santiago, FONIS, 2007.

García, M.; Torres, M. P. \& Ballesteros, E. Enfermería geriatrica. Barcelona, Masson, 2002.

Henry, R. G., \& Ceridan, B. Delivering dental care to nursing home and homebound patients. Dent. Clin. North Am., 38(3):537-51, 1994.

Instituto Nacional de Estadísticas (INE). Población y Sociedad. Aspectos demográficos. Santiago, Gobierno de Chile, 2008. Disponible en: http://www.ine.cl/canales/ chile_estadistico/demografia_y_vitales/demografia/pdf/ poblacion_sociedad_enero09.pdf

Kandelman, D.; Gagnon, G.; Hurley, R. \& Ruel, D. Elderly dental care. Needs and costs in three different resident settings. Oral Health, 80(4):73-6, 1990.

Manderson, R. D. \& Ettinger, R. L. Dental status of the institutionalized elderly population of Edinburgh. Community Dent. Oral Epidemiol., 3(3):100-7, 1975.

Ministerio de Planificación y Coordinación (MIDEPLAN). Encuesta de Caracterización Socioeconómica Nacional (CASEN). Santiago, Gobierno de Chile, 2009. Disponible en: www.mideplan.cl

Misrachi, C. \& Espinoza, I. Utilidad de las mediciones de la calidad de vida relacionada con la salud. Rev. Dent. Chile, 96(2):28-35, 2005.

Misrachi, C.; Sepúlveda, H. \& Lamadrid, S. Situación protésica y conductas asociadas en adultos mayores de nivel socioeconómico medio-alto y bajo. Rev. Dent. Chile, 93(1):10-6, 2002.

Morishita, M.; Takaesu, Y.; Miyatake, K.; Shinsho, F. \& Fujioka, M. Oral health care status of homebound elderly in Japan. J. Oral Rehabil., 28(8):717-20, 2001.
Nathanson, C. A. Illness and the feminine role: a theoretical review. Soc. Sci. Med., 9(2):57-62, 1975.

Paunovich, E. Assessment of the oral health status of the medically compromised homebound geriatric patient: a descriptive pilot study. Spec. Care Dentist, 14(2):80-2, 1994.

Petersen, P. E.; Bourgeois, D.; Ogawa, H.; Estupinan-Day, S. \& Ndiaye, C. The global burden of oral diseases and risks to oral health. Bull. World Health Organ., 83(9):6619, 2005.

Ritchie, C. S.; Burgio, K. L.; Locher, J. L.; Cornwell, A.; Thomas, D.; Hardin, M. \& Redden, D. Nutritional status of urban homebound older adults. Am. J. Clin. Nutr., 66(4):815-8, 1997.

Servicio Nacional del Adulto Mayor (SENAMA). Estudio de situación de los adultos mayores dependientes con pensión asistencial y sus cuidadores domiciliarios. Santiago, Gobierno de Chile, 2007. Disponible en: http:// w w w. s e n a m a.cl/file s a p p / Estudio_de_situacion_de_AM_con_pension_ y_cuidadores_domiciliarios.pdf

Servicio Nacional del Adulto Mayor (SENAMA).. Las personas mayores en Chile. Situación, avances y desafíos del envejecimiento y la vejez. Santiago, Gobierno de Chile, 2009. Disponible en: http://www.senama.cl/ filesapp/las_personas_mayores_en_ chile_situacion_avances_y_desafios_2.pdf

Tada, A.; Watanabe, T.; Yokoe, H.; Hanada, N. \& Tanzawa, $\mathrm{H}$. Oral bacteria influenced by the functional status of the elderly people and the type and quality of facilities for the bedridden. J. Appl. Microbiol., 93(3):487-91, 2002.

United Nations (UN). World Population Ageing. New York, United Nations, 2009. Disponible en: http://www.un.org/ esa/population/publications/WPA2009/WPA2009report.pdf

Wolrd Health Organization (WHO). Oral Health Surveys: Basic Methods. 4th ed. Geneve, World Health Organization, 1997.

Dirección para Correspondencia:

María E. Quinteros

Departamento de Salud Pública

Facultad de Ciencias de la Salud

Universidad de Talca

Talca

Recibido : 05-08-2013

CHILE

Aceptado: 10-09-2014

Email: mariaelisaquinteros@gmail.com 